\title{
علامة تشكيل جديدة لتسهيل الاختصار العلمي في اللغة العربية
}

\section{A new mark (haraka) in Arabic to facilitate medical and scientific abbreviations}

\author{
Khaled Moustafa ${ }^{1}$ خالد مصطقى
}

ملخص

تُعاني اللغة العربية، مقارنة مع اللغات ذات الجذور اللاتينية، من ندرة المصطلحات الطبية والعلمية، لعدم وجود طربقة سهلة ومعتمدة للاختصار العلمي والطبي بما بتلاعم مع طبيعة وخصائص اللغة العربية. لذلك، سيتم هنا اقتراح إدخال علامة أو شَرْطَة عمودية جديدة إلى اللغة العربية للمساعدة على تبسيط الاختصار والاثتقاق العلمي من خلا دمج الحروف الأولى من كلمات أو عبارات عربية أطول، ثم وضع علامة عمودية على الحرف الأخير من الكلمة الناتجة، لتعريفها على أنها اشتقاق علمي متخصص لإزالة أي إبهام أو غموض حول لفظها الغربب أو معناها، الذي قد يكون مبهماً أو غامضاً أو مجهولاً. مثال: يمكن اختصار عبارة "ضِغط دِم مِرتفع" ب: ضدم'، بدمج الحروف الأولى ووضع الثَّرْطَة العمودية بُعَيْد الحرف الأخير "الميم" من الكلمة الجديدة ضدم' للالالة على أنها اشتقاق علمي بحت، دالّته الثّزّطة العمودية في آخر الكلمة. وهذه طريقة سهلة نسبياً يمكن أن تساعد على إثراء القاموس العلمي العربي بالمصطلحات والاشتقاقات العلمية الجديدة في شتى الميادين لحل معضلة ندرة المصطلحات العلمية وصعوبة تركيبها أو اشتقاقها في اللغة العربية.

\begin{abstract}
Arabic language suffers from a paucity of medical and scientific terms/acronyms compared with Latin languages, due to the absence of clear, easy and reliable method for scientific and medical abbreviation. A new method will be proposed here to facilitate scientific abbreviations in Arabic by the introduction of a new diacritical mark (haraka) as a short vertical dash (') to be placed at the last letter of an Arabic acronym to label it as a specialized medical or scientific term that can be recognized in more details in its field of specialization. The proposed method is simple, easy and should help enrich the Arabic dictionary with specialized medical and scientific nomenclature in various disciplines.
\end{abstract}

\section{Résumé}

La langue arabe souffre d'un manque évident de termes/acronymes scientifiques et médicaux par rapport aux langues latines, en raison de l'absence de méthode claire, simple et fiable pour abréger

\footnotetext{
${ }^{1}$ Editor of Arabic Science Archive (https://arabixiv.org). Email: khaled.moustafa@arabixiv.org محرر الأرشيف العربي العلمي
} 
Arabic Science Archive (arabixiv.org) | 2019

et adopter des termes scientifiques. Dans cet article, une nouvelle méthode simple sera proposée pour faciliter l'abréviation scientifique en arabe par l'introduction d'un nouvel accent (haraka) sous forme d'un tiret vertical (') à placer au niveau de la dernière lettre d'un acronyme arabe afin de l'identifier comme un terme médicale ou scientifique spécialisé. L'approche est simple et facile à mettre en œuvre pour enrichir le dictionnaire arabe avec de la nomenclature médicale et scientifique dans différents domaines.

تتميز اللغات ذات الجذور اللاتينية بسهولة الاشتقاق والاختصار اللغوي بسبب طريقة الكتابة بأحرف منفصلة، وطربقة لفظ الاختصار كما هي في غير الاختصار، في كثير من الحالات. فلو أخذنا مثلاً مصطلح "الحمض النووي الريبوزي منقوص الأوكسجين deoxyribonucleic acid" المعروف اختصاراً ب DNA نجد أن لفظ الاختصار DNA (دي إن إيه) هو نفسه لفظ الحروف التي تكونه عند قراءتها منفصلة (D, N, A).

يسمح الاختصار في اللغة بتوفير بعض وقت الكتابة والقراءة ومساحة الطباعة وتقصير اللفظ وتسهيله، إذا كانت العبارات الأصلية طوبلة نوعاً ما وصعبة اللفظ والحفظ. وبتذمر كثير من الدارسين العرب في المجال الطبي والعلمي من اللغة العربية وينعتونها بالصعوبة والعجز عن الاثتقاق والاصطلاح، واستيعاب المفردات والتراكيب العلمية الحديثة، ومواكبة التطورات العلمية المتسارعة.

ولكن هذه المزاعم غير صحيحة، لأن اللغة العربية هي أداة تواصل مطواعة، وقابلة للاشتقاق والاختصار والإدغام، وتتسم بالمرونة التي تجعلها ملائمة لمواكبة التطورات العلمية والاختصارات والاشتقاقات الطبية والتخصصية. فقد مرت اللغة العربية بكثير من المراحل، بدءاً من الكتابة بحروف منفصلة إلى إدخال التتقيط والحركات، أو علامات النشكيل المختلفة، للتكيّف مع أوضاع لغوبة جديدة رافقت انتشار اللغة العربية إلى أمصارٍ جديدة لا يتقن أهلها بالضرورة اللغة العربية جيداً. وتضم علامات النتكيل في اللغة العربية ما يلي: 1) الكسرة (-) وهي خط صغير مائل يوضع تحت الحرف لإضافة صوت باء قصير إليه. 2) الضمة (ُ)): وهي بمثابة حرف واو صغير يُكتب فوق الحرف لإضافة صوت واو قصير إليه. 3) الفتحة (-): وهي خط مائل شبيه بالكسرة ولكن يكتب فوق الحرف لإضافة صوت ألف قصير إليه. 4) التتوين: وهو مضاعفة الحركات الثالث أعلاه (الكسرة والضمة والفتحة) لإضافة لفظ النون إلى آخر الحرف المنوّن.

5) السكون ( ) : وهو حلقة أو دائرة صغيرة مغلقة توضع فوق الحرف لإسكان أو جزم اللفظ عنده لِبرهة. 6) الثدّة (هّ): وهي حركة تشبه رقم ثلاثة متجه للأعلى لنتديد أو مضاعفة اللفظ للحرف الذي تُوضع فوقه. 
ويؤدي استخدام هذه الحركات إلى تسهيل اللفظ وتمييز المعنى، خاصة عند وجود تثابه بالكلمات والحروف، أو لتعليم الأطفال كيفية النطق الصحيح للحروف، أو لمن يتعلم العربية من غير الناطقين بها.

\section{علامة عمودية جديدة لتسهيل الاختصار العلمي}

يمكن الاعتماد على خصوصية اللسان العربي بوجود علامات النشكيل أعلاه، بإدخال علامة أخرى جديدة كعلامة اشتقاق أو اختصار أو نحت تساعد على تسهيل الاشتقاق والاختصار العلمي والطبي. لذا، أقترح هنا أن يتم إدخال علامة تتكيل مستحدثة على شكل خط مسنقيم عمودي قصير أو شرطة عمودية ( ' ) توضع فوق الحرفين الأول والأخير من كلمة أو عبارة مختصرة أو مشتقة من عبارة أطول للالالة على أنها اشتقاق علمي، مع لثمديه إمكانية إهمال

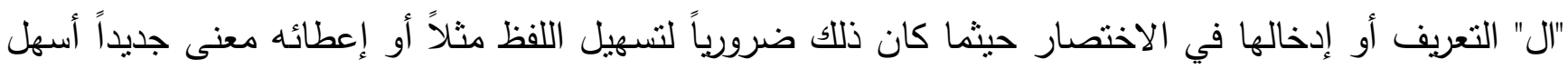

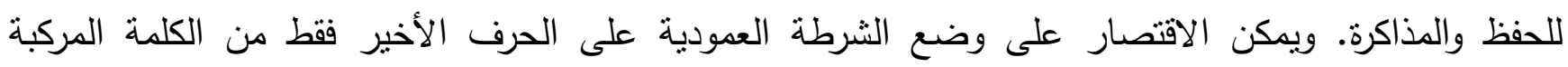

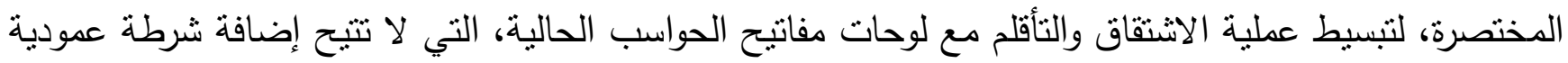

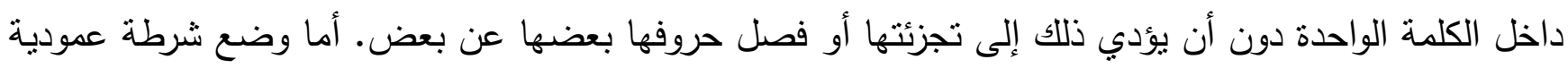

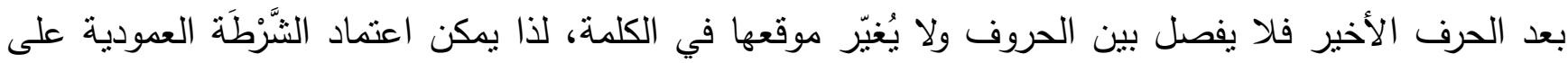
الحرف الأخير فقط للالالة على أن كلمة ما هي مصطلح أو تعبير علمي متخصص بمكن رده إلى أصله لفهم

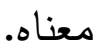

لنأخذ على سبيل المثال كلمة DNA المذكورة آنفاً، وهي اختصار للعبارة الإنكليزية deoxyribonucleic acid وتعني بالعربي "الحمض النووي الريبي منقوص الأوكسجين"، وهي معرّبة في كثير من المراجع العربية بـ "دنا"،

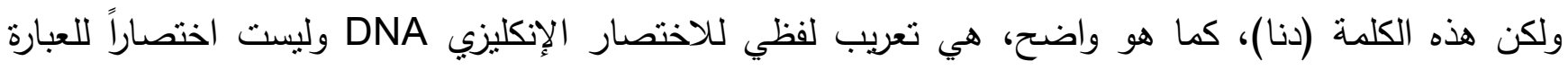

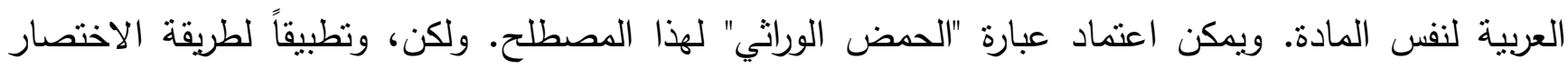
المقترحة هنا باستخدام شرطة عمودية، يمكن دمج الحروف الأولى من العبارة العربية الكاملة، ثم وضع الثَرْطَة

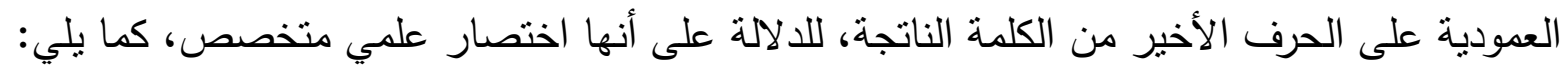
العبارة العربية الكاملة: الحمض الـووي الرِيبي مـققوص إلأوكسجين

الاختصار العربي لهذه العبارة، تبعاً للطريقة المقترحة هنا، بإهمال "ال" التعريف في الكلمات الأولى، ووضع شرطة عمودية بعد آخر حرف من الكلمة المركبة الناتجة عن الامج، هو : حنرما' - التي يمكن لفظها بالتتكيل: حَنَرْمَا' يكفي، والحال هذه، أن يُلاحظ القارئ وجود الثرطة العمودية فوق الحرف الأخير لكي يتم التعرف على أن الكلمة الغريبة اللفظ والمعنى هنا (حنرما') هي اختصار علمي لإزالة اللبس والاستغراب حول معناها أو دلالتها التي ستكون مبهمة وغير مألوفة للكثيرين. 
وفيما يلي بعض الأمتلة الأخرى عن الاختصار وفق هذه الطريقة، بوضع الثَّزُطَة العمودية على الحرف الأخير من

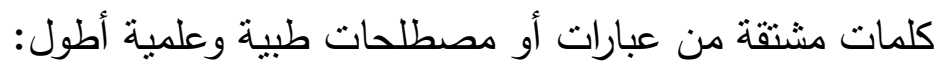

ضِغط دِم مِرتقع (hypertension): ضدم'

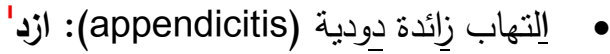

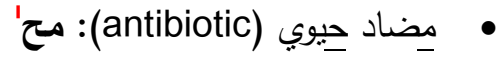

• • أغذية معدلة وراثياً (genetically modified food): أمو'

نحليل طيفي بالرِنين المغناطيسي (magnetic resonance spectroscopy): تطرم'

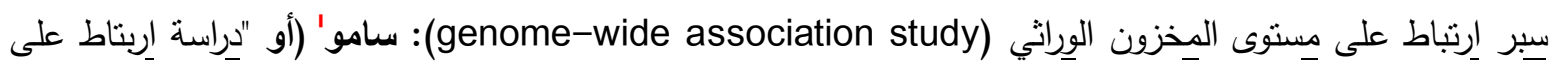

مِنتوى المخزون الوِراثي"): دامو' (بإدغام حرفي الميح المتتاليين في كلمتي "مستوى و "مخزون" في حرف ميم واحد)

إلخ. ويمكن تجريبها على مصطلحات أخرى من مختلف التخصصات.

لا شك أن لفظ الكلمات الناتجة سيكون غريباً للوهلة الأولى وغير معهود، كما هو الحال بالنسبة للأمتلة هنا

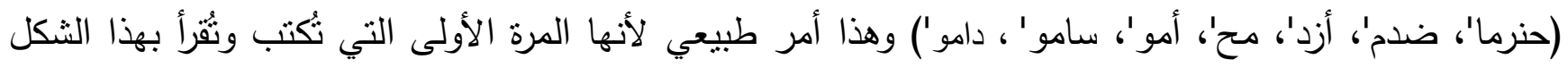

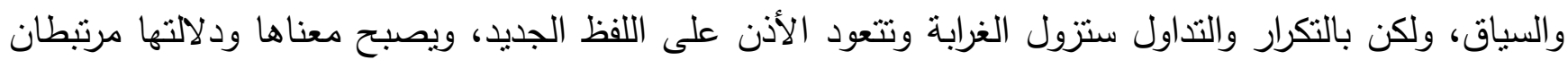
بالعبارات التي تتنتق منها، أو المادة التي تتشير إليها (مثلاً، كلمة حنرما' سنتشير إلى المادة الوراثية الموجودة في

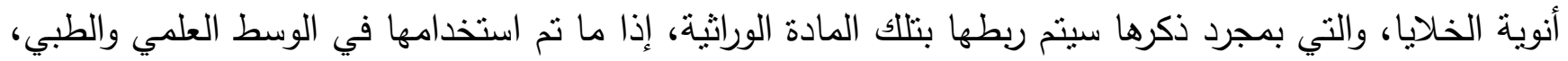
كأي مصطلح علمي آخر يتم تداوله باستمرار). وهكذا، بالنسبة إلى أب مصطلح علمي آخر في أب مجال، لأن التكرار هو وسيلة الأُلفة.

أما فيما يتعلق بطريقة لفظ الكلمات المشتقة بهذه الطريقة، فيمكن أن يخضع للسليقة اللغوية التي تجمع بين سلاسة

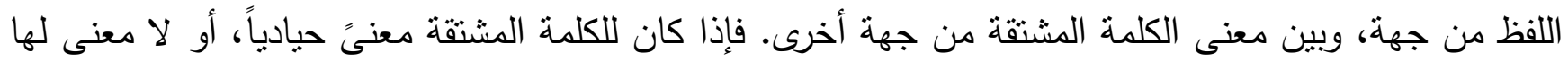

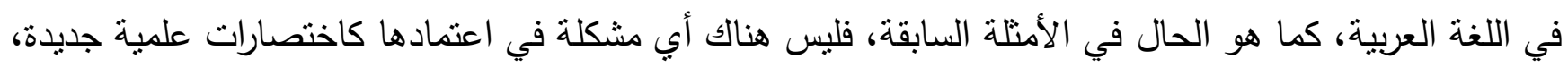

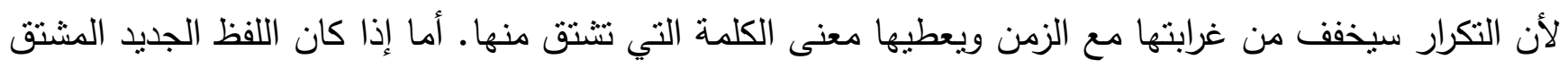
ازدرائياً أو رذيلاً، أو ذا صبغة سلبية أو معنيً مذموماً، فيمكن اختيار حروف أخرى تلي الحروف الأولى في العبارة

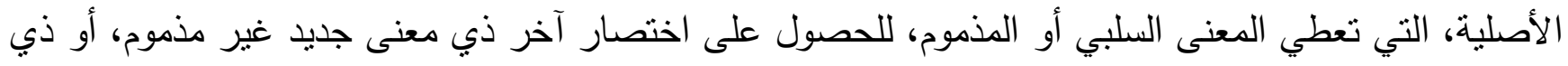
معنى حيادياً غير مألوف أو غير معروف من قبل.

وهكذا، سيكون وجود شَرَطَة عمودية فوق الحرف الأخير لكلمة ما بمثابة علامة مُميّزة للتعرف على أي كلمة على أنها اختصار علمي بحت، يمكن البحث عنه أو الرجوع إلى أهل الاختصاص لمعرفة كنهه بدقة إذا كان مبهماً أو

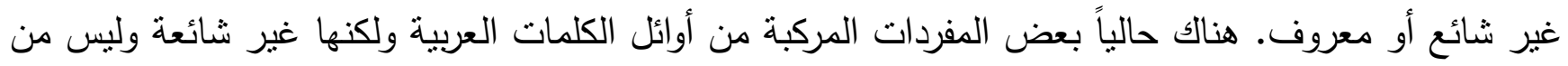


السهولة التعرف عليها كمصطلحات أو اختصارات علمية، لعدم وجود أي إثارة أو طريقة تدل على ذلك. لذا، فإن وجود علامة عمودية في أواخر الكلمات المشتقة بهذه الطريقة يمكن أن يحل مشكلة الإبهام أو الغموض حول لفظها

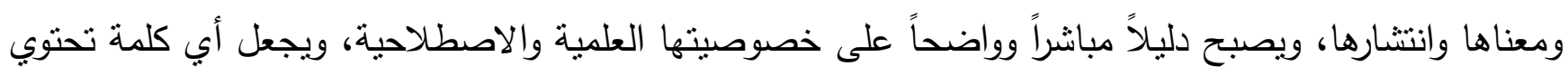
شَرْطَة عمودية في آخرها مصطلحاً علمياً مشتقاً من عبارات وأطول.

ويمكن أن تساهم هذه الطريقة في تكوين وترسيخ مفردات علمية جديدة بطريقة سهلة ومبسطة، وإثراء القاموس

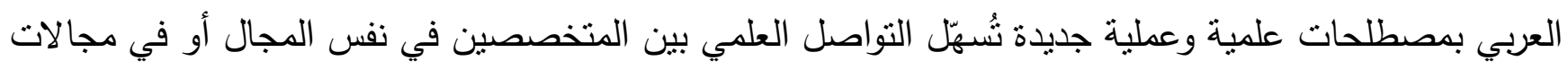

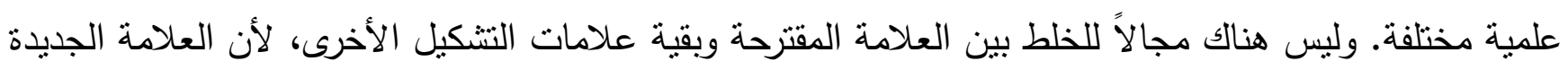

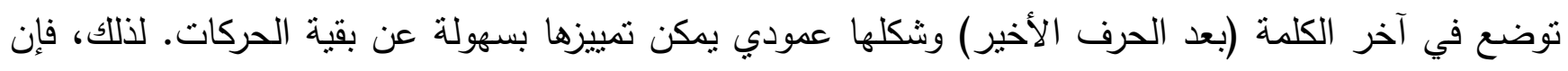
وجود شرطة عمودية فوق الحرف الأخبر سيكون علامة خاصة بالاختصارات والمصطلحات الطبية والعلمبة فقط.

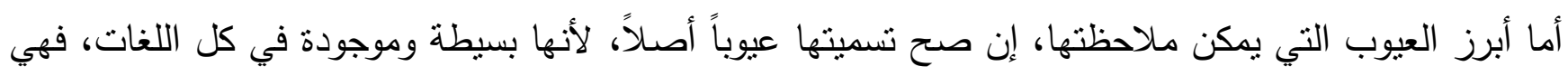

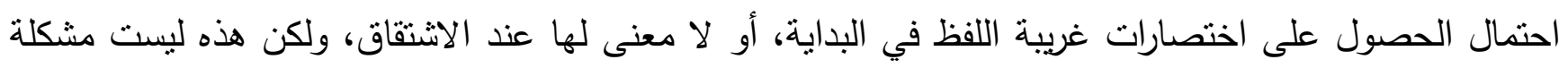

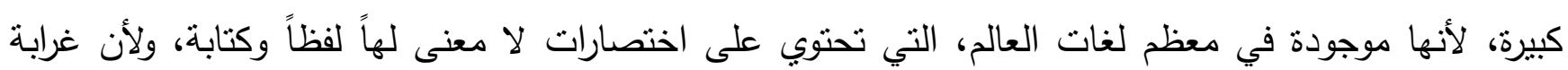

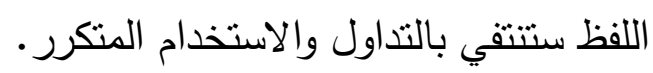

ختاماً، على الرغم من أن الاختصار والاشتقاق والإدغام ليست شروطاً إجباربة في الكتابة العلمية لأي لغة، إلا أنها أساليب شائعة وكثيرة الاستخدام في النصوص العلمية الحديثة المكتوبة باللغات ذات الجذور اللاتينية. بالمقابل، تعاني اللغة العربية من ندرة المصطلحات العلمية لأسباب كثيرة أهمها عدم وجود طريقة سهلة تئطر الاثتقاق العلمي وتضع له قواعد واضحة وقابلة للنطبيق على نطاق واسع. لذا، يمكن أن نساهم الطريقة المقترحة هنا، بدمج الحروف الأولى من عبارات علمية طويلة ثم استخدام شرطة عمودية توضع في نهاية الكلمة الجديدة، في حل

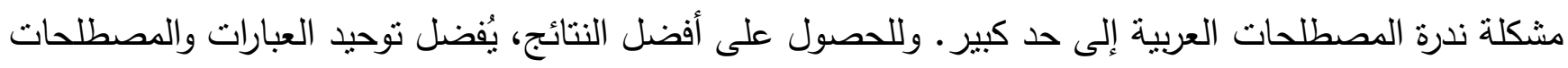
العربية قبل الاختصار لإنتاج مشتقات علمية موحدة بين الدول العربية تُسهّل التواصل العلمي والتدريسي وتقلل التعارض والاختلاف الاصطلاحي، علماً أن تللك ليست مشكلة كبيرة أيضاً، طالماً أنه يمكن التعرف على لئل العبارات الاشتقاقية من خلال الثَّزْطَة العمودية التي ستوضع في نهاية الكلمة المشتقة كمصطلح جديد يمكن اعتماده في

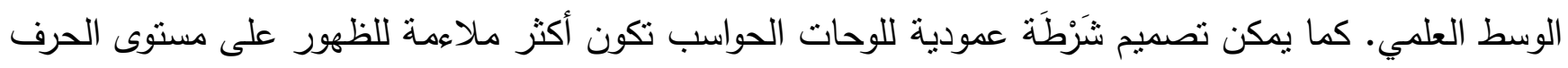

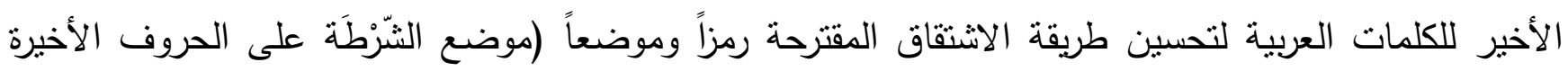
للكلمات العربية دون أن تتعارض مع موقع وشكل بقية الحركات). كما يمكن أن تخضع المفردات المكونة بهذه

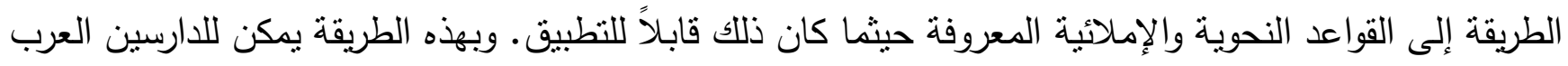
في أي مجال علمي أو تقني تكوين مصطلحات مهنية وتخصصية بسهولة ويسر كلٌ في مجاله. 
ويمكن طبعاً اعتماد بعض أو كثير من الاختصارات المعربة لفظياً لعبارات شائعة عالمياً، واستخدامها كما هي، ولكن إذا ما تم اعتماد جميع تلك الاختصارات في أي لغة، فإن تلك اللغة ستفتقد خصوصيتها ويتضاءل عدد مفرداتها. لذلك، لا بد من إيجاد طريقة للاختصار العلمي والاشتقاق والتأقلم بما يتلاءم مع طبيعة كل لغة وخصوصياتها.

كلمات دلالية: اللغة العربية والاشتقاق العلمي، مصطلحات علمية عربية، اثتقاق علمي، علامات النتكيل العربية، حركات التتكيل العربية، دمج الكلمات العربية، دمج المصطلحات العلمية، النحت في اللغة العربية، الاختصار العلمي في اللغة العربية 